\title{
Visual Experience-Dependent Maturation of Correlated Neuronal Activity Patterns in a Developing Visual System
}

\author{
Heng Xu, ${ }^{1}$ Arseny S. Khakhalin, ${ }^{2}$ Arto V. Nurmikko, ${ }^{1,3}$ and Carlos D. Aizenman ${ }^{2}$ \\ ${ }^{1}$ Department of Physics and ${ }^{2}$ Department of Neuroscience, and ${ }^{3}$ Division of Engineering, Brown University, Providence, Rhode Island 02912
}

The functional properties of neural circuits become increasingly robust over development. This allows circuits to optimize their output in response to a variety of input. However, it is not clear whether this optimization is a function of hardwired circuit elements, or whether it requires neural experience to develop. We performed rapid in vivo imaging of calcium signals from bulk-labeled neurons in the Xenopus laevis optic tectum to resolve the rapid spatiotemporal response properties of populations of developing tectal neurons in response to visual stimuli. We found that during a critical time in tectal development, network activity becomes increasingly robust, more correlated, and more synchronous. These developmental changes require normal visual input during development and are disrupted by NMDAR blockade. Our data show that visual activity and NMDAR activation are critical for the maturation of tectal network dynamics during visual system development.

\section{Introduction}

Developing neural circuits become increasingly reliable over time. As neural circuits mature, responses of neurons within the circuit become more robust and less variable, often generating specific and repeatable spatiotemporal patterns of activity (Beggs and Plenz, 2004; Ikegaya et al., 2004). These changes are caused in part by maturation of both synaptic and intrinsic properties of individual neurons, but also by fine-tuning of the connectivity patterns within the neurons in a network (Schulz, 2006; Pratt et al., 2008; Maffei and Fontanini, 2009). For example, in the optic tectum of Xenopus laevis tadpoles, neural responses to visual stimuli are known to strengthen over development (Pratt and Aizenman, 2007), and visual responses become increasingly reliable because of refinement of local tectal circuitry (Pratt et al., 2008). Stimulus selectivity and receptive field size are also known to refine over development in both zebrafish embryos and Xenopus tadpoles (Niell and Smith, 2005; Tao and Poo, 2005; Dong et al., 2009). However, it is not clear whether these changes in network reliability require neural activity or not. There is ample evidence suggesting that many functional properties of neural circuits require patterned sensory activity to develop normally (Cline, 1998; Crair, 1999; Ruthazer and Aizenman, 2010), yet there is also significant evidence that several properties can de-

Received Nov. 4, 2010; revised March 18, 2011; accepted April 12, 2011.

Author contributions: H.X., A.S.K., A.V.N., and C.D.A. designed research; H.X. and A.S.K. performed research; A.V.N. contributed unpublished reagents/analytic tools; H.X., A.S.K., and C.D.A. analyzed data; H.X. and C.D.A. wrote the paper.

This work was supported by National Science Foundation (NSF) Division of Integrative Organismal Systems, the National Eye Institute of the National Institutes of Health, and the Whitehall Foundation (A.S.K. and C.D.A.); and NSF Grants EFRI-BSBA 0937848 and ECS-0423566 (H.X. and A.V.N.). We thank Elie Bienenstock, David Sheinberg, and Barry Connors for helpful discussion and advice. We also thank Edward Ruthazer for helpful advice regarding the manuscript.

Correspondence should be addressed to Carlos D. Aizenman, Brown University, Department of Neuroscience, Box G-LN, Providence, RI 02912. E-mail: Carlos_Aizenman@brown.edu.

DOI:10.1523/JNEUROSCI.5802-10.2011

Copyright $\odot 2011$ the authors $\quad 0270-6474 / 11 / 318025-12 \$ 15.00 / 0$ velop normally in the absence of sensory input (Niell and Smith, 2005; Komai et al., 2006; Nevin et al., 2008; Golshani et al., 2009).

In this study, we focus on the development of spatiotemporal activity patterns in optic tectal neuronal networks of Xenopus tadpoles. In the Xenopus optic tectum, visual responses result both from direct activation of tectal neurons by retinal ganglion cell (RGC) axons and from activation by local intratectal circuits (Pratt et al., 2008). The timing of these network responses becomes more reliable over development. Although temporally patterned visual activity has been shown to entrain the timing of visual responses in the tectum over a period of a few hours, it is unknown whether the long-term maturational changes in the reliability of visual responses require sensory experience during development. Furthermore, it is not known whether developmental changes in visual response timing result from plasticity at local synapses within a single neuron or whether they reflect a broader reorganization of the entire tectal network.

To address these questions, we used bulk-loading of $\mathrm{Ca}^{2+}$. sensitive dyes in combination with rapid image acquisition to detect large-scale patterns of neural activity across large populations of tectal neurons in response to visual stimuli. By using a deconvolution algorithm on the $\mathrm{Ca}^{2+}$ fluorescence signal, we were able to detect synchronous neural spiking across neurons with a high temporal resolution. We first describe the development of neural synchrony and correlated activity between developmental stages 46 and 48/49, a time period where a substantial amount of growth, reorganization, and functional change occur within the tectal circuit (Tao and Poo, 2005; Akerman and Cline, 2006; Pratt et al., 2008; Dong et al., 2009). We then test whether normal development requires visual experience and NMDA receptor-mediated activity.

\section{Materials and Methods}

Animal rearing conditions. All animal experiments were performed in accordance with Brown University Institutional Animals Care and Use Committee approved animal protocols. Wild-type Xenopus laevis tad- 
poles were raised in $10 \%$ Steinberg's solution [ $1 \times$ Steinberg's (in mM): 10 HEPES, $\left.58 \mathrm{NaCl}, 0.67 \mathrm{KCl}, 0.34 \mathrm{Ca}\left(\mathrm{NO}_{3}\right)_{2}, 0.83 \mathrm{MgSO}_{4}, \mathrm{pH} 7.4\right]$ in $19-21^{\circ} \mathrm{C}$ incubators until developmental stage 46 or stage $48 / 49$ (typically $8-10 \mathrm{~d}$ ). The sex of the tadpoles cannot be determined at this stage of development. Animals were kept on a $12 \mathrm{~h}$ light/dark cycle in the normal rearing conditions. Some animals were maintained in a dark chamber to block any light between stages 46 and 48/49. Another experimental group was exposed to $10 \mu \mathrm{M}$ NMDAR blocker MK-801 (Tocris Bioscience) between stages 46 and 48/49.

Animal dissection. Tadpoles were anesthetized in $0.01 \%$ (v/v) MS-222 (Sigma) and pinned to a $11 \mathrm{~cm}$ imaging chamber filled with $4 \mathrm{ml}$ of HEPES-buffered extracellular saline $(115 \mathrm{~mm} \mathrm{NaCl}, 6 \mathrm{~mm} \mathrm{KCl}, 3 \mathrm{~mm}$ $\mathrm{CaCl}_{2}, 0.5 \mathrm{~mm} \mathrm{MgCl}_{2}, 5$ mм HEPES, $10 \mathrm{~mm}$ glucose, $\mathrm{pH}$ 7.25, $255 \mathrm{mOsm}$ ) containing $100 \mu \mathrm{M}(+)$-tubocurarine (Sigma) for animal immobilization. We carefully pierced and removed the skin covering the brain, exposing the surface of the tectum. A cut through the dorsal commissure was then made to expose the optic tectal lobes, and the medial surface was flattened to facilitate optical imaging (Aizenman et al., 2003; Dong et al., 2009). For the isolated whole-brain preparation, brains were filleted along the dorsal midline and removed from the body. Brains were pinned down onto a block of Sylgard in the imaging chamber (Aizenman et al., 2003; Pratt et al., 2008). For both in vivo recordings and whole-brain recordings, the ventricular membrane was vacuumed off with a broken micropipette tip before the dye loading to help dye diffusion and penetration into the tissue. All recordings were performed at room temperature $\left(20-22^{\circ} \mathrm{C}\right)$.

Calcium indicator loading and calcium imaging. OGB1-AM (Invitrogen) was dissolved at a concentration of $2 \mathrm{~mm}$ in DMSO with $10 \%$ pluronic acid (Invitrogen) and diluted to a final concentration of $10 \mu \mathrm{M}$ in the extracellular saline in the imaging chamber. Samples (either the in vivo preparation or the whole-brain preparation) were incubated with this solution for $45 \mathrm{~min}$ for dye staining and subsequently washed with fresh extracellular saline three times. The imaging chamber was then mounted on the stage of a Nikon E600FN upright microscope with a $40 \times$ objective and an OGB-1 filter set (Semrock) for imaging. Excitation light coming from a high-power blue LED (LXK2-PB14-N00; Philips Lumileds Lighting) through the back port of the microscope illuminated the neurons, generating green fluorescence, which was projected on the CCD surface of a high-speed neural imaging camera (NeuroCCD SMQ; Redshirt Imaging). The OEM software Neuroplex (Redshirt Imaging) was used for image acquisition and basic analysis. An imaging area of $100 \times$ $100 \mu \mathrm{m}$ with $80 \times 80$ pixels was achieved at a frame rate of 125 frames per second (fps). The imaging time was set to $4 \mathrm{~s}$ for evoked-response recordings (in vivo preparations) and $16 \mathrm{~s}$ for spontaneous recordings (wholebrain preparations).

For each experiment, $\sim 30-90$ fluorescent tectal neurons were imaged on the same field of view. The total number of cells imaged was not significantly different across experimental conditions. Ten trials with identical conditions were recorded for each group of cells. A minimum of 10 experiments was collected for each developmental stage (stages 46 and 48/49) and different experimental conditions (dark-reared animals and MK-801-treated animals).

Visual stimulation. In the evoked response recordings, whole-field visual stimuli were generated by a custom-built blue LED (illumination peak: $470 \mathrm{~nm}, 30 \mathrm{~mA}$ current injection), which was coupled directly to an optic fiber (500 $\mu \mathrm{m}$ core diameter) for light guidance. The fiber end was mounted on a MP-285 micromanipulator (Sutter Instruments) for positional control. The distance between the fiber end and the tadpole's eye was $<200 \mu \mathrm{m}$. The LED was turned on $2 \mathrm{~s}$ after the start of the imaging trial and remained on for $1 \mathrm{~s}$.

Electrophysiology. Visualized cell-attached recordings of fluorescent tectal cells were made with micropipettes $(8-12 \mathrm{M} \Omega$ ) filled with K-gluconate-based intracellular saline (100 mm K-gluconate, $8 \mathrm{~mm} \mathrm{KCl}$, $5 \mathrm{~mm} \mathrm{NaCl}, 1.5 \mathrm{~mm} \mathrm{MgCl}_{2}, 20$ mm HEPES, 10 mм EGTA, 2 mM ATP, and $0.3 \mathrm{~mm}$ GTP, pH 7.25, $255 \mathrm{mOsm})$. Signals were measured with a Axoclamp 2B amplifier (Molecular Devices), digitized at $10 \mathrm{kHz}$ using a Digidata 1440A A-D board and acquired using p-Clamp 10 software (Molecular Devices). Data were analyzed together with imaging data using a custom-written Matlab (Mathworks) program.
For whole-cell recordings, recordings were performed using a multiclamp 700B amplifier (Molecular Devices) under the same recording conditions as above. To find the onset latency, each trace was fit with a function, as follows: $f=-\max \left\{0, a_{1} \cdot \exp \left[-b_{1}(t-x)\right]-a_{2}\right.$. $\left.\exp \left[-b_{2}(t-x)\right]\right\}$. Then, latencies of these idealized responses were calculated as follows: lat $=x+\log \left(a_{1} / a_{2}\right) /\left(b_{1}-b_{2}\right)$.

Spike train reconstruction from calcium-imaging data. Neuronal spike trains were reconstructed from fluorescence changes resulting from changes in intracellular calcium levels. Although calcium signals have a slow decay time, there are several methods to detect rapid increases in fluorescence that correspond to neuronal spiking (Ikegaya et al., 2004; Niell and Smith, 2005; Yaksi and Friedrich, 2006; Sasaki et al., 2008). Here we used a deconvolution algorithm to reconstruct periods of neuronal spiking from the calcium-imaging data.

For each optical recording, regions of interest (ROIs) were drawn to cover the fluorescent neuronal cell bodies. Each ROI typically was comprised of $\sim 40$ pixels. Averaged intensity signals of each ROI were exported into text files from the Neuroplex software for further analysis using custom-written Matlab programs.

The change of fluorescence intensity of a ROI was calculated as

$$
\frac{\Delta F}{F}=\frac{F-F_{0}}{F_{0}},
$$

where $F$ is the averaged intensity of the ROI in an image frame and $F_{0}$ is the fluorescence baseline, chosen as the minimal averaged fluorescence level (over every 15 consecutive frames) of the ROI over the recording.

The normalized data were then filtered three times using a boxcar filter (window size: 7 frames) to smooth out small fluctuations most likely caused by shot noise. The filtered signals were then deconvolved with a kernel based on a unitary calcium transient kernel: $y=e^{-t / 5 \mathrm{~s}}-e^{-t / 0.2 \mathrm{~s}}$. This kernel is the result of fitting calcium transients resulting from single spikes, as measured electrophysiologically.

For calibration purposes, a subset of tectal neurons $(>5$ neurons for each developmental stage and rearing condition) were loose-patched to record action potentials while simultaneously being imaged. $\mathrm{Ca}^{2+}$ responses resulting from single spikes were averaged to generate a deconvolution kernel for each experimental condition. Each spike (or burst) measured electrophysiologically was correlated with the peak of the deconvolved waveform of the calcium signal. The relationship between the number of spikes in a burst and the amplitude of the corresponding deconvolution peak was plotted to generate a calibration curve for each developmental stage and rearing condition (Fig. 1). Responses with different numbers of spikes (including single spikes) produced peak amplitudes with different ranges, and a group of threshold values [ $\operatorname{th}(N)$, with $N$ representing the spike number; e.g., 1, 2, 3, 4, etc.] was set to infer the spike number of a burst from its corresponding deconvolution peak. Optimization was made on these thresholds to allow the best prediction with minimal error for each developmental stage and rearing condition.

To predict the timing of individual spikes from a deconvolved trace, we first determined the relationship between the actual spike onset latency and the latency to the peak of the deconvolved trace by comparing these two measures. Although the two latencies were closely related, they did not match exactly. We were able to correct this discrepancy by using a complementary measure to compensate for the difference. The latency difference between the spike onset and the peak of the deconvolution peak was found to be linearly proportional to the width of the deconvolved trace peak. Plotting one versus the other provided a curve by which we could accurately predict spike timing from the deconvolved trace. Thus, prediction of the spike timing (or the onset timing of a burst) was made based on both the peak latency and peak width of the deconvolved trace, yielding an error of $<10 \mathrm{~ms}$. To predict the timing of individual spikes within a burst, we used the simultaneously recorded electrophysiological data to determine the relationship between interspike interval and the spike number in a burst, and plotted this relationship for each developmental stage and rearing condition (Fig. 1). The spike frequency during a burst is linearly related to the number of spikes in a train, such that trains with more spikes have shorter interspike intervals, whereas bursts with fewer spikes have longer interspike 
A

B

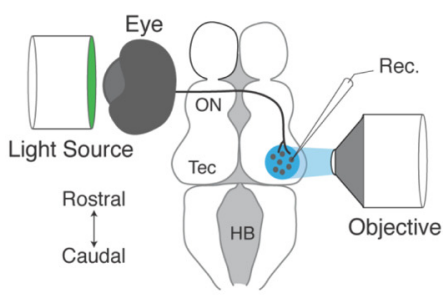

$\mathrm{D}$

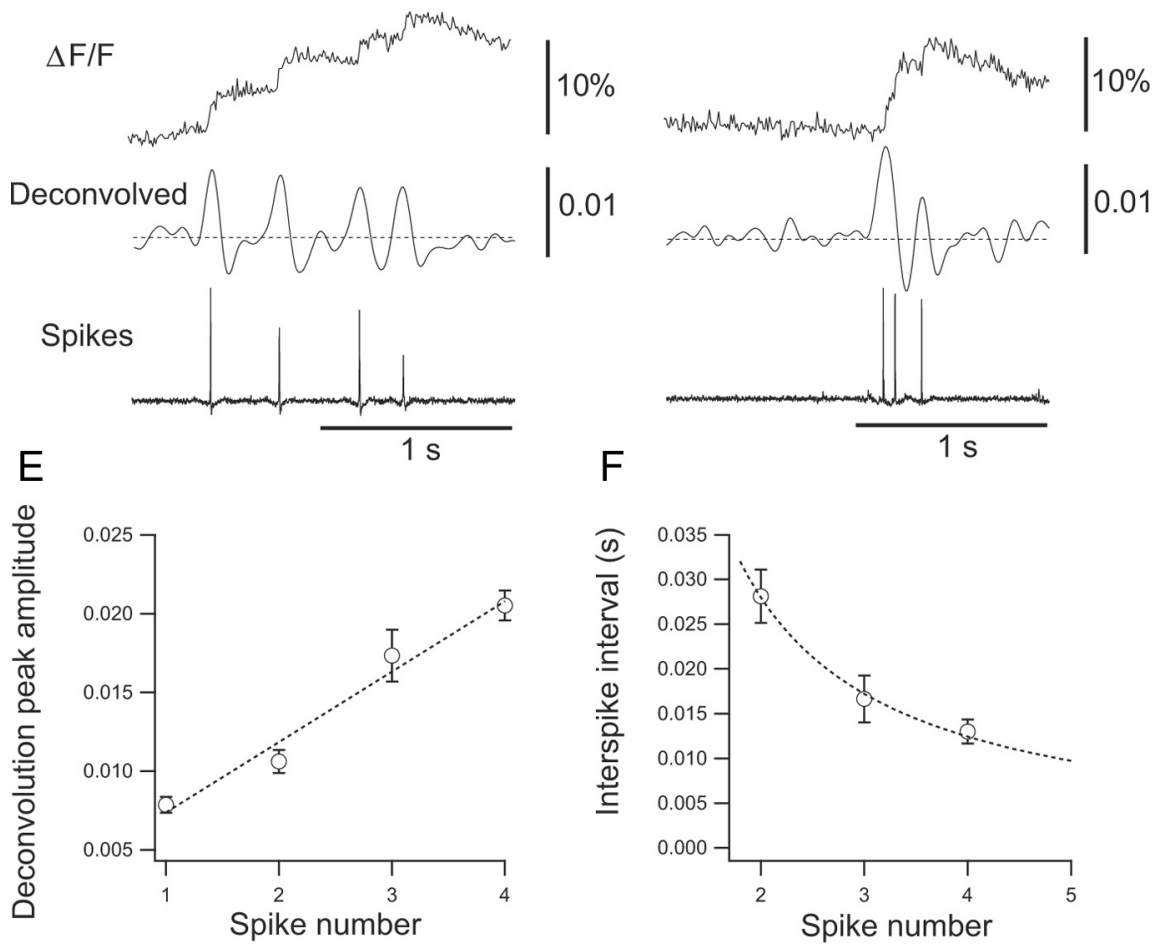

Figure 1. High-speed calcium imaging of visual responses in tadpole optic tectum in vivo. A, Schematic of the experimental setup. Fiber-optic-guided whole-field visual stimuli were projected onto the tadpole's eye, while visual responses in calcium dye-loaded neurons in the contralateral tectum were imaged using a high-speed CCD camera. $\boldsymbol{B}$, A fluorescence image of bulklabeled tectal neurons in the surface of the optic tectum. $\boldsymbol{C}$, Sample calcium traces of selected tectal neurons ( $\boldsymbol{B}$, circles) in response to a visual stimulus. The timing of visual stimulus is represented by the bottom blue bar. $\boldsymbol{D}$, Two examples of data analysis performed on the fluorescence traces. Top, Raw fluorescence traces from two separate dye-loaded neurons in response to a visual stimulus. Middle, Deconvolved fluorescence traces, showing multiple peaks; $y$-axis represents amplitude of the deconvolved trace (see Materials and Methods). Bottom, Simultaneous electrical recording using loose-patch electrodes shows that spikes correspond to peaks in the deconvolved trace. $\boldsymbol{E}$, Relationship between the peak amplitude of the deconvolved trace and the number of spikes measured electrophysiologically (linear fit, data from stage 46 animals). $\boldsymbol{F}$, Relationship between the interspike interval and the number of spikes during a burst of spikes (data fitting using inversely linear relations, data from stage 46 animals). From this information, we can reconstruct the timing of individual spikes from the fluorescent signal generated by the calcium-sensitive dyes in response to a visual stimulus. Error bars are SEM. Rec., Recording; Tec, tectum; HB, hindbrain.

intervals. Thus, we used an inverse linear function to predict the interspike interval within a burst and, by extension, the total duration of the spike burst (Fig. $1 F$ ).

Using the above calibration curves for determining the number of spikes, spike or burst onset, and burst duration from deconvolved traces, we reconstructed spike trains from the calcium-imaging data. Thresholds $d_{\mathrm{N}}$ were first applied to the deconvolved calcium signals for the detection of spikes (or bursts). The spike number of each burst was predicted from the corresponding deconvolution peak height, and the spike timing (or the onset of a burst) was estimated from the deconvolution peak timing and peak width. The slow decay of calcium transients does not allow the detection of precise timing of each spike in a burst; how- ever, the precise timing of each spike is not important for the calculation of cross-correlations values, as described below. Therefore, spike trains were reconstructed as square pulses of spikingfrequency profiles (using the interspike interval inference mentioned above) instead of trains of individual spikes, with its area equal to the predicted number of spikes.

Data analysis. The reconstructed spike trains were then used to compute the amount of cross-correlation between individual cell pairs within a population. The cross-correlation coefficient of a cell pair was calculated based on the formula

$$
Q_{\rho \sigma}(\tau)=\left|\begin{array}{|l}
\int \rho(t) \sigma(t+\tau) d t \\
\frac{\tau}{\tau} \rho^{2}(t) d t \cdot \int_{0}^{\tau} \sigma^{2}(t) d t
\end{array}\right|,
$$

with $\rho$ and $\sigma$ representing the reconstructed spike train, and the angle brackets representing the average over different recordings. The maximal value of $Q_{\rho} \sigma(\tau)$ over different $\tau$ was used for statistical analysis.

To calculate trial-to-trial variability within individual cells, we computed the trial-to-trial correlation of cell activity over different trials, which was calculated as

$$
Q_{\rho \rho^{\prime}}=\left|\begin{array}{|l}
\int \rho(t) \rho^{\prime}(t) d t \\
\int_{0}^{\tau} \rho^{2}(t) d t \cdot \int_{0}^{\tau} \rho^{\prime 2}(t) d t
\end{array}\right|,
$$

with $\rho$ and $\rho^{\prime}$ representing the different recordings of the same cell and the angle brackets representing the average over different recording pairs.

The Pearson product-moment correlation coefficient was calculated according to the following definition from Rodgers and Nicewander (1988):

$$
r_{X Y}=\frac{\langle(X-\bar{X})(Y-\bar{Y})\rangle}{\left\langle(X-\bar{X})^{2}\right\rangle^{1 / 2}\left\langle(Y-\bar{Y})^{2}\right\rangle^{1 / 2}} .
$$

Custom-written Matlab programs were used for all data analysis. All remaining statistical tests were done using InStat Prism or GraphPad using nonparametric methods (Mann-Whitney).

Computational model. A two-layer network model with period boundary conditions was used and was primarily based on Song et al. (2000) and Song and Abbott (2001). The output layer was composed of 100 integrate-and-fire neurons following a standard implementation, where the membrane potentials obey

$$
\tau_{\mathrm{m}} \frac{d V}{d t}=V_{\text {rest }}-V+\sum g_{\mathrm{ex}}\left(E_{\mathrm{ex}}-V\right)+\sum g_{\text {in }}\left(E_{\text {in }}-V\right),
$$


with $\tau_{\mathrm{m}}=20 \mathrm{~ms}, V_{\text {rest }}=-74 \mathrm{mV}, E_{\text {ex }}=0 \mathrm{mV}$, and $E_{\text {in }}=-74 \mathrm{mV}$. Each synaptic input is modeled as an instantaneous conductance increase ( $g_{\text {exm }}$ or $\left.g_{\text {inm }}\right)$ in response to an input spike, with exponential decay, where $g_{\text {ex }}(t)=g_{\text {exm }} \exp \left(-t / \tau_{\text {ex }}\right)$ and $g_{\text {in }}(t)=g_{\text {inm }} \exp \left(-t / \tau_{\text {in }}\right)$, with $\tau_{\text {ex }}=$ $\tau_{\text {in }}=5 \mathrm{~ms}$. The threshold for firing an action potential was set to -54 $\mathrm{mV}$, and the membrane potential was reset to $-60 \mathrm{mV}$ after firing.

The input layer contained 500 neurons that fired through Poisson processes in response to a point stimulus:

$$
r_{\mathrm{a}}=R_{0}+R_{1}\left(e^{-(s-a)^{2} / 2 \sigma^{2}}+e^{-(s+500-a)^{2} / 2 \sigma^{2}}-e^{-(s-1000-a)^{2} / 2 \sigma^{2}}\right)
$$

with $R_{0}=2 \mathrm{~Hz}, R_{1}=25 \mathrm{~Hz}$ [based on data from tadpole retinal recordings (J. Demas and H.T. Cline, unpublished observations) ], $\sigma=50$, and $s$ and $a$ being the position of the stimulus and the input layer neuron, respectively (three terms in the brackets preserved the periodicity). The point stimulus $s$ was chosen randomly between 1 and 500 from a uniform distribution and kept stable for a time interval chosen from an exponential distribution with a mean of $\tau_{\mathrm{r}}=50 \mathrm{~ms}$.

Each output layer neuron received excitatory synaptic inputs from input layer neurons and other output layer neurons, as well as from an additional background Poisson input at $500 \mathrm{~Hz}$ through a synapse of strength 0.096, which mimicked the effects of a large number of afferents.

The spike-timing-dependent plasticity (STDP) rule was modeled as a change of the synaptic strength $g_{\text {exm }}=g_{\text {exm }}+g_{\max } F(\Delta t)$ for the corresponding presynaptic and postsynaptic spike pair, where $\Delta t=t_{\text {pre }}-t_{\text {post }}$ and

$$
F(\Delta t)=\left\{\begin{array}{cc}
A_{+} \exp \left(\Delta t \tau_{+}\right), & \Delta t<0 \\
-A_{-} \exp \left(\Delta t \tau_{-}\right), & \Delta t \geq 0
\end{array}\right.
$$

with $g_{\max }=0.015, A_{+}=0.0003, \tau_{+}=\tau_{-}=20 \mathrm{~ms}$ for feedforward connections and $40 \mathrm{~ms}$ for recurrent connections. To avoid uncontrolled synaptic growth and to be certain that feedforward connections dominated recurrent connection, the ratio of the areas under the negative and positive portions of the STDP window function was set to be $B=A_{-} \tau_{-} /$ $A_{+} \tau_{+}=1.06$ for feedforward connections and 1.04 for recurrent connections. $g_{\text {exm }}$ was set to change between 0 and $g_{\max }$ and would set to the appropriate limit value once reaching the boundary.

The initial feedforward connection strengths were set to be

$$
0.15 g_{\max } \exp \left(-\frac{1}{2}\left(\frac{d_{i j}}{50}\right)^{2}\right),
$$

where $d_{i j}=\min (|i /(5-j)|, 100-|i /(5-j)|)$ for input layer neuron $i$ and output layer neuron $j$. The recurrent connections between output layer neurons $(j, k)$ were limited in the range $\min (|j-k|, 100-|j-k|) \leq 25$, and were set initially to 0 . All-to-all inhibitory connections with $g_{\text {inm }}=$ $0.15 g_{\max }$ were also applied to the recurrent network.

After the STDP-based development step, the resulting network activity was compared with the initial network by applying whole-field stimuli $\left(r_{\mathrm{a}}=R_{0}+R_{1}\right.$ for all input layer neurons; $\tau_{\mathrm{r}}=100 \mathrm{~ms}$ ) multiple times. A time delay of the stimuli was randomly chosen each time from a normal distribution with $\bar{t}_{\text {delay }}=0.1 \mathrm{~s}$ and $\sigma_{\text {delay }}=0.02 \mathrm{~s}$. Spike trains of 50 consecutive output layer neurons were saved for $1 \mathrm{~s}$ and filtered three times using a boxcar filter (window size: $56 \mathrm{~ms}$ ). The remaining data analysis steps were the same as the experimental data process. Simulated spontaneous activity was acquired by generating and saving output layer activity during a $16 \mathrm{~s}$ simulation period with no stimuli $\left(r_{\mathrm{a}}=0\right)$.

\section{Results}

\section{Fast $\mathrm{Ca}^{2+}$ imaging of large neuronal populations}

To measure changes in neural synchrony among tectal neurons during development, we measured simultaneously the intracellular $\mathrm{Ca}^{2+}$ levels of multiple tectal neurons in response to a whole-field visual stimulus. The intracellular $\mathrm{Ca}^{2+}$ concentration, recorded using $\mathrm{Ca}^{2+}$-sensitive fluorescence indicators, has been used as an indirect measure of spiking activity of neurons previously (Yuste et al., 2000; Badea et al., 2001; Cossart et al.,
2003; Ikegaya et al., 2004; Niell and Smith, 2005; Ramdya et al., 2006; Yaksi and Friedrich, 2006; Nikolenko et al., 2007; Sasaki et al., 2008). Using a deconvolution algorithm, in combination with a fast sampling rate, these $\mathrm{Ca}^{2+}$ responses can be used to predict spike latencies with a high degree of accuracy (Yaksi and Friedrich, 2006; Sasaki et al., 2008). In this study, we exposed tectal neurons, whose cell bodies are located on the surface of the tectum, to a membrane-permeant $\mathrm{Ca}^{2+}$ indicator (Oregon Green BAPTA-AM) for 45 min before washing the indicator out from the extracellular media using artificial CSF. This resulted in a substantial number of tectal neurons brightly labeled by the indicator (for details, see Materials and Methods, above). Animals were then imaged on a recording chamber with a high-speed CCD camera at a rate of $125 \mathrm{fps}$. Compared with conventional two-photon imaging, our method allows for much higher temporal resolution without losing spatial resolution, allowing us to better resolve spatiotemporal activation patterns across several tectal neurons. Whole-field flashes were used as visual stimuli and were delivered using a custom-made blue LED coupled to a $500 \mu \mathrm{m}$ optic fiber that was placed close $(<200 \mu \mathrm{m})$ to the tadpole's eye (Fig. $1 \mathrm{~A})$. An area $\sim 100 \times 100$ $\mu \mathrm{m}$ with 30-90 labeled neurons in the contralateral tectum was imaged (Fig. $1 \mathrm{~B}$ ). Visual stimuli, consisting of $1 \mathrm{~s}$ on and $1 \mathrm{~s}$ off, were delivered after $2 \mathrm{~s}$ following the onset of the optical recording. Only the neuronal activity occurring within the on period was analyzed (Fig. 1C).

Visual stimuli evoked spiking activity in tectal neurons, which was accompanied by rapid increases in fluorescence corresponding to an increase in intracellular $\mathrm{Ca}^{2+}$. The fluorescence signal then decayed slowly back to resting levels with a time constant of $\sim 1$ s. To find the relationship between the fluorescence signal and neuronal spiking, a subset of tectal neurons were loosepatched to record action potentials electrophysiologically while simultaneously imaging $\mathrm{Ca}^{2+}$ signals. By assuming that each spike generates a similar $\mathrm{Ca}^{2+}$ fluorescence transient, which is then linearly added to the existing fluorescence signal, the fluorescence signals $(\Delta F / F)$ were deconvolved using a kernel derived from $\mathrm{Ca}^{2+}$ transients resulting from single spike events (for details, see Materials and Methods, above). The resulting traces were then compared with the electrophysiological traces of the same cell and each individual spike was found to closely correspond to a peak of the deconvolved trace (Fig. 1D). For a highfrequency spike burst, the peak of the deconvolved trace was larger and its height correlated with the number of spikes in the burst. Figure $1 E$ shows the averaged results from multiple $(n=7)$ stage 46 neurons and demonstrates that the relation between the deconvolution peak height and the spike number is approximately linear. The interspike interval during a burst of spikes was found to be inversely proportional to the number of spikes in the burst, allowing us to predict the total duration of a burst from the calculated number of spikes (for details, see Materials and Methods, above) (Fig. 1F). Thus, we were able to decode the relationship between the fluorescence signal and the electrical activity of the cell, both during single spikes and highfrequency bursts. This allowed us to translate the fluorescence signals back to electrical signals with a high degree of accuracy (timing error: $0-16 \mathrm{~ms}$; spike number error: $<1$ ). For each developmental stage and imaging condition, we empirically determined a separate calibration curve to compensate for any differences in $\mathrm{Ca}^{2+}$ signaling and intrinsic excitability across groups. 


\section{Development of correlated evoked neural activity}

Using this deconvolution method, we reconstructed the electrophysiological activity of groups of tectal neurons in response to whole-field visual stimuli. In each experiment, we measured the activity of a population of tectal neurons. Only cells that responded reliably to the stimulus (i.e., $>50 \%$ of the time) were included in the analysis so as to eliminate the possibility of recording from unhealthy neurons. The total number of cells that met this criteria in each experiment was not significantly different across experimental conditions. We first looked at the development synchronous responses across neurons in a population by measuring the degree of correlation of neural activity between cells. This was achieved by calculating the average peak crosscorrelation coefficient of each pair of cells over 10 trials (for details, see Materials and Methods, above) and then plotting the distribution of average correlation values for each experiment. Bigger correlation coefficients represent more consistent and coherent neural activity, which may be important for more reliable information processing. Comparisons were made between developmental stages 46 and 48/49 tadpoles, covering a period in development during which substantial changes in tectal cell intrinsic excitability, synaptic transmission, receptive field properties, and visually guided behavior are known to occur (Aizenman et al., 2003; Tao and Poo, 2005; Pratt and Aizenman, 2007; Dong et al., 2009). We found a significant increase in the correlation coefficient between stages 46 and 48/49, as seen in the correlation maps and correlation coefficient histograms shown in Figure $2 A, B$. The averaged results across multiple experiments ( $n=9$ for stage 46 and $n=14$ for stage $48 / 49$ ) show the shift of the correlation coefficient distribution (Fig. 2C) and a significant increase in the mean cross-correlation coefficient between the two groups (stage 46: $0.220 \pm 0.019, n=9$; stage 48/49: $0.74 \pm 0.036, n=14 ; p<0.0001$ ) (Fig. 2D). This indicates that tectal neural response patterns become significantly more coherent and precise over development.

This developmental increase in neural correlation is consistent with previous studies showing that during development, retinotectal projections become stronger and more focused (Cline, 1998; Ruthazer and Cline, 2004) and excitatory tectal recurrent connections become refined (Pratt et al., 2008), both of which may help increase the accuracy of the relative response timing across neurons observed in this study. These synaptic changes are known to be experience-dependent and some involve NMDA receptor activation (Ruthazer and Cline, 2004; Pratt et al., 2008). To test whether the increases in correlated activity observed here are also dependent on visual experience, we compared normally reared stage 48/49 tadpoles with tadpoles darkreared between developmental stages 46 and 48/49 (7-9 d old) (for details, see Materials and Methods, above). Strikingly, we found that dark rearing completely eliminates the developmental increase in neural correlation, leaving the correlation distribution almost unchanged compared with stage 46 (Fig. $2 A-C$ ), with a low mean cross-correlation coefficient $(0.224 \pm 0.021, n=17$; $p=0.859$ ) (Fig. 2D). To test whether the increase in correlation required NMDA receptor activation, tadpoles were reared in NMDAR blocker MK-801 (10 $\mu \mathrm{M})$. In contrast to dark rearing, blockade of NMDA receptors only partially disrupted development, resulting in a more flat distribution of correlation coefficients (Fig. $2 A-C$ ) and a smaller change in the mean crosscorrelation coefficient $(0.524 \pm 0.025, n=17)$ (Fig. $2 D)$. Although MK-801 did not completely block developmental changes in neuronal correlation, the changes were significantly smaller than in control stage $48 / 49$ tadpoles $(p<0.0001)$. The
A
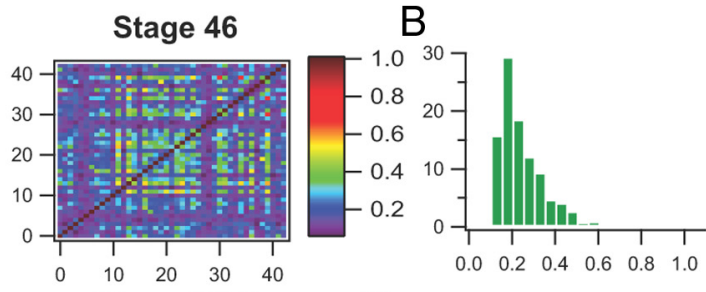

Stage $48 / 49$
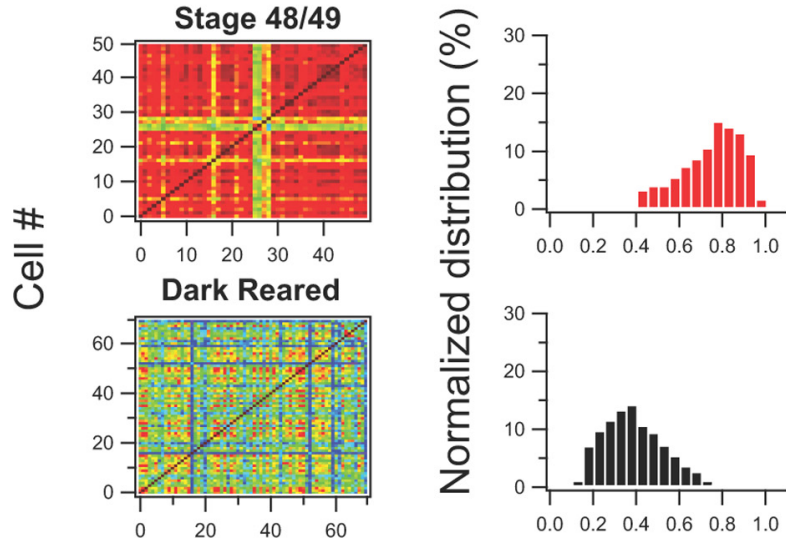

MK-801
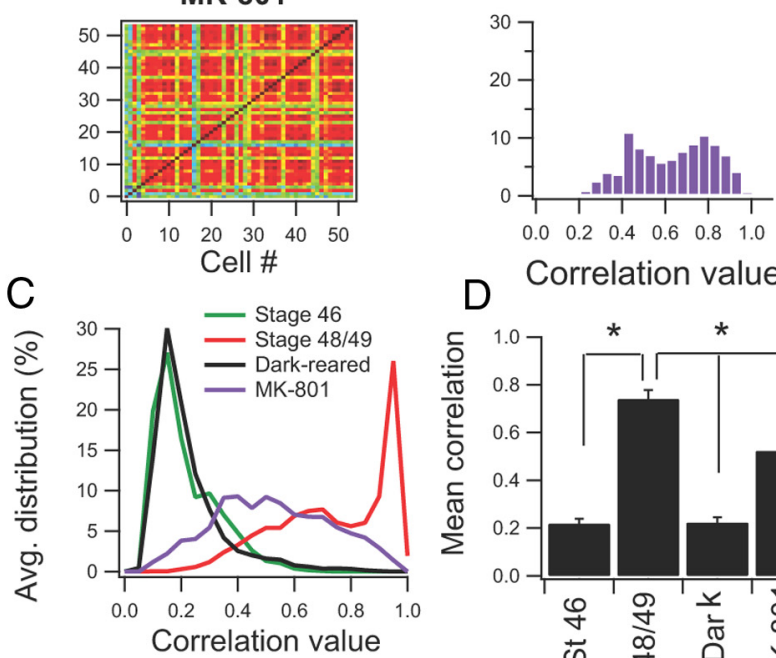

D

Correlation value

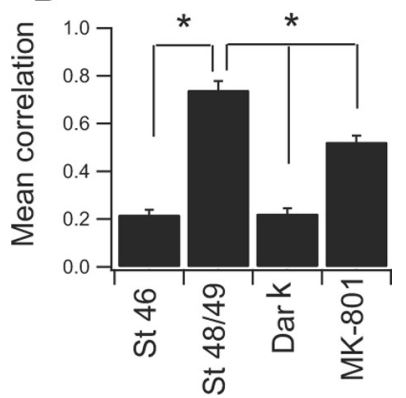

Figure 2. Developmental increase in correlated neural activity among large groups of tectal neurons is dependent on visual experience and NMDAR activation. $\boldsymbol{A}$, Correlation matrix for pairs of tectal neurons bulk labeled with $\mathrm{Ca}^{2+}$ indicator dyes in response to a whole-field visual stimulus. Colors represent average correlation values for each cell pair over 10 trials. One sample experiment is shown for each experimental condition. The matrix contains no spatial information, thus cells near each other in the matrix are not necessarily spatially near each other in the tectum. $\boldsymbol{B}$, Normalized distribution of correlation values for pairs of tectal neurons from the same example as in $\boldsymbol{A}$. $\boldsymbol{C}$, Averaged distributions of correlation values across multiple experiments for each experimental condition. Notice the overall shift to higher correlation values between stages 46 and 48/49, as well as the effect of dark rearing and MK-801 treatment in preventing this shift. $\boldsymbol{D}$, Average of total mean correlation value for each developmental stage and rearing conditions. ${ }^{*} p<0.05$; see text for exact $p$ values and number of experiments. Error bars are SEM.

incomplete effect of MK-801 on development is likely due to the fact that multiple mechanisms of plasticity, such as those requiring activation of $\mathrm{Ca}^{2+}$-permeable AMPARs, also contribute to circuit refinement (Pratt et al., 2008). Alternatively, the effect of MK-801 could be due to a partial reduction in total synaptic drive, resulting in an intermediate effect. In either case, our findings support the hypothesis that normal activity is required for proper development of correlated activity. 
In a previous study (Pratt et al., 2008), we showed that tectal cell spiking evoked by direct optic nerve stimulation became more temporally compact over development. This previous study was done with synaptic inhibition blocked and reflected a reorganization of local circuits. We checked whether the same trend was observed under the more physiological conditions used in this study - in vivo visual stimulation with intact inhibition. Using cell-attached recordings, we found that over development, spike patterns became more temporally compact and the median spike latency decreased (median latency, stage 46: $581 \pm 106 \mathrm{~ms}, n=6$; stage 49 : $126 \pm 29 \mathrm{~ms}, n=7, p=0.001$; train duration (10-90 percentile), stage 46: $328 \pm$ $88 \mathrm{~ms}, n=6$; stage $49: 132 \pm 26 \mathrm{~ms}, n=7$, $p=0.044)$. This trend was disrupted by dark rearing and partially disrupted by MK-801 (median latency, dark: $321 \pm 66$ $\mathrm{ms}, n=8, p=0.024$; MK-801: $258 \pm 51$ ms, $n=7, p=0.044$; train duration (10-90 percentile), dark: $490 \pm 52 \mathrm{~ms}$, $n=8, p<0.001$; MK-801: $510 \pm 72 \mathrm{~ms}$, $n=7, p<0.001)$. This confirms that even under physiological conditions, we see a significant temporal refinement of spike responses over development, which is dependent on normal visual activity. Further, it shows that this temporal reorganization is likely contributing to the increase in correlated activity observed during development.

Together, these findings suggest that as a result of visual experience between stages 46 and $48 / 49$, the amount of correlated activity among tectal neurons significantly increases. This refinement is caused, at least in part, by activation of NMDAR.

Development of visual-response timing To further examine the development of temporally coherent responses between tectal neurons, we analyzed the onset latencies of visual responses across neurons. Most neurons responded to light a few hundred milliseconds after the onset of the visual stimulus (Fig. 3A). Stage 48/49 animals showed a much shorter mean onset latency (averaged over 10 trials for each cell, and then averaged across cells for each experiment) compared with stage 46 animals (stage 46: $0.29 \pm 0.04 \mathrm{~s}, n=9$; stage 48/49: $0.17 \pm 0.02 \mathrm{~s}, n=14 ; p=0.023$ ) (Fig. $3 D$ ). This observation is consistent with a developmental increase in the strength of retinotectal synapses. Dark-reared stage 48/49 tadpoles had similar onset latency values as stage 46 animals $(0.37 \pm$ $0.03 \mathrm{~s}, n=17$ ) (Fig. $3 D$ ) and were significantly slower than control stage $48 / 49$ tadpoles ( $p<0.0001$ ), possibly due to abnormal development of retinotectal inputs, resulting in weak synaptic connections. Rearing in MK-801 resulted in an intermediate effect, consistent with our previous finding $(0.27 \pm 0.02 \mathrm{~s}, n=17)$
A

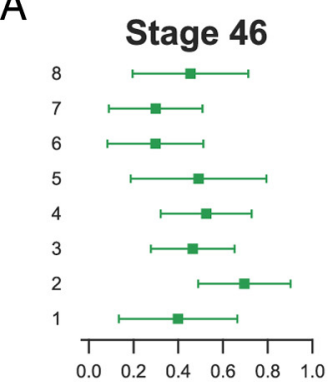

B $\quad$ C
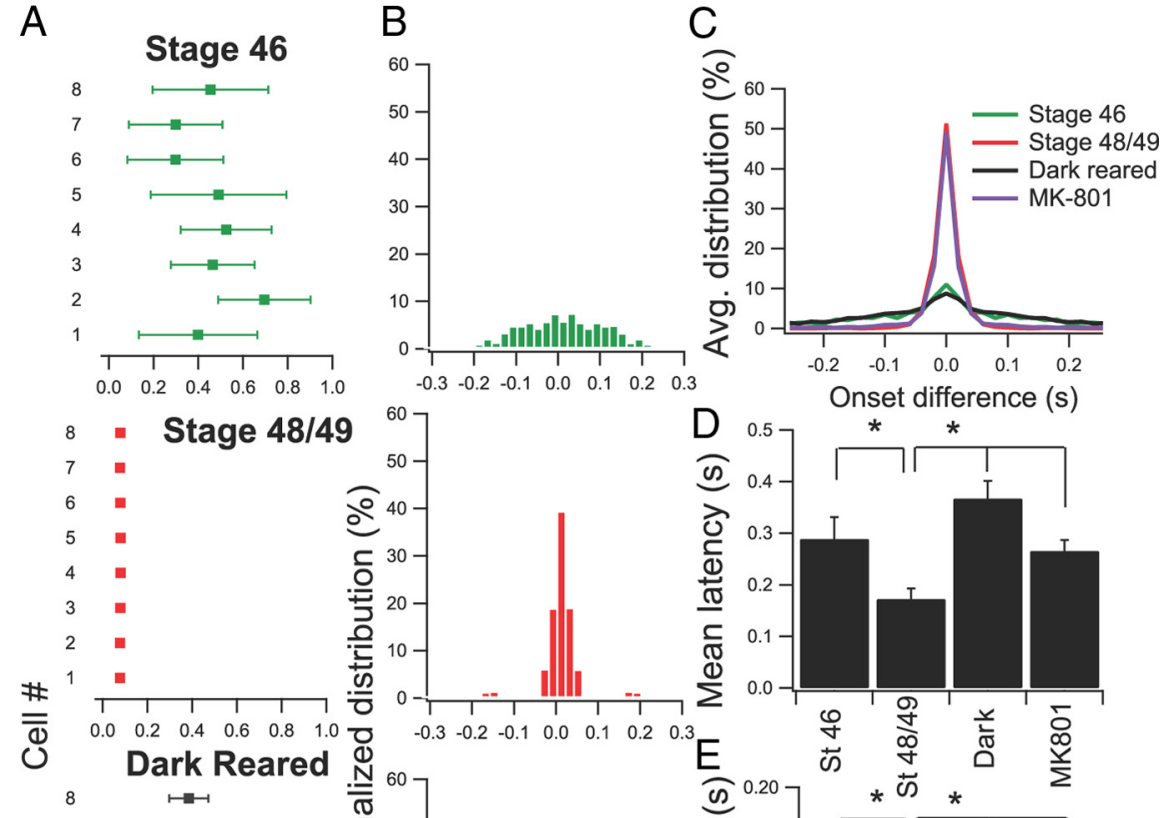

를

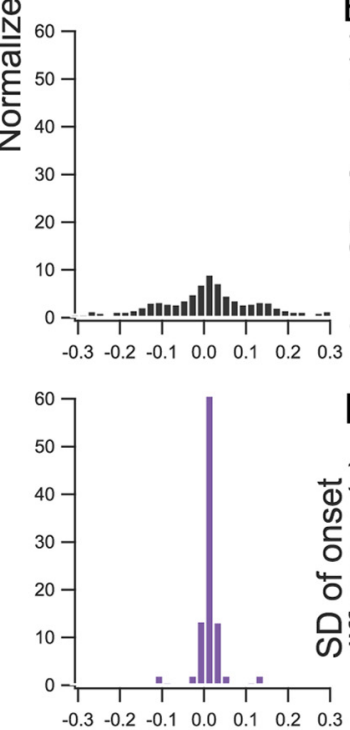

Onset latency (s)

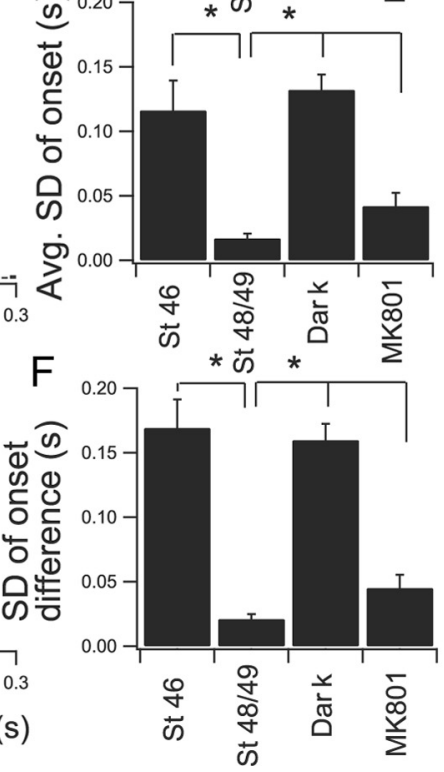

Figure 3. Developmental increase in visual response synchrony among tectal neurons. $\boldsymbol{A}$, Examples of visual response onse latencies from different developmental stages and rearing conditions. Each example illustrates response onset latencies from eight randomly selected tectal neurons in the same recording. Error bars indicate variability over 10 trials. $\boldsymbol{B}$, Sample distributions of visual response onset latency differences between pairs of tectal neurons. One example is shown for each experimental group. $\boldsymbol{C}$, Averaged normalized distributions of response onset latency differences for each experimental group. Notice the narrowing of the response onset differences between cells over development, indicating increased synchronous activation. D, Average mean visual response onset latencies for each experimental group. $\boldsymbol{E}$, Averaged SDs of visual response onset latencies for each experimental group. For each experiment, the SD of the onset latencies across trials was calculated for each cell; these values were averaged to obtain the mean value for each experiment. This measure indicates the variability in response onsets across trials. $\boldsymbol{F}$, Averaged SD onset latency differences for each experimental group. The SD of onset latency differences was calculated from the values in $\boldsymbol{B}$ for each experiment and reflect the width of the distribution. Less synchronous activation will result in higher values. ${ }^{*} p<0.05$; see text for exact $p$ values and number of experiments. $\boldsymbol{D}-\boldsymbol{F}$, Error bars are SEM. St, Stage.

(Fig. 3D); this group was also significantly slower than control stage $48 / 49$ tadpoles ( $p=0.0031$ ). We also looked at variability in the onset latency across trials for individual cells by measuring the SD of the onset latency (over 10 trials for each cell) (Fig. 3A). We then averaged the SDs across different cells in a given experiment to get an index of total response onset variability for that tectum. We next compared this measure across experimental conditions. This measure was found to drop dramatically between stages 46 and 48/49 (stage 46: $0.12 \pm 0.02 \mathrm{~s}, n=9$; stage 48/49: $0.02 \pm 0.004$ 
s, $n=14 ; p=0.002$ ) (Fig. 3E), indicating that retinotectal activation becomes more reliable. This trend was completely prevented by dark rearing $(0.13 \pm 0.01 \mathrm{~s}, n=17 ; p<0.0001)$ (Fig. $3 E$ ), whereas MK-801 treatment resulted in a much smaller effect but still significantly different from stage $48 / 49$ controls ( $0.04 \pm$ $0.01 \mathrm{~s}, n=17 ; p=0.0265$ ) (Fig. 3E).

The onset latency differences between cell pairs were also measured as another metric to describe the level of neural synchrony between cells. We first calculated the average onset latency difference for each cell pair in a given experiment (for more details, see Materials and Methods, above) and plotted the distribution of onset difference values (Fig. 3B). We then averaged the normalized distributions for each developmental stage and rearing condition (Fig. 3C). Developmental stage 48/49 tadpoles had a much narrower distribution of onset latency differences, as evidenced by a significantly smaller SD compared with stage 46 tadpoles (stage 46: $0.17 \pm 0.02 \mathrm{~s}, n=9$; stage 48/49: $0.02 \pm 0.004 \mathrm{~s}, n=14 ; p<$ 0.0001 ) (Fig. $3 F$ ), representing the development of more synchronized neural activity. Tadpoles reared in the dark displayed similar distributions and mean value as stage 46 animals and were very significantly different from normal-reared stage $48 / 49$ animals (0.16 \pm $0.01 \mathrm{~s}, n=17 ; p<0.0001$ ) (Fig. 3F). The MK-801-treated group, in contrast, showed a more modest yet significant disruption of development $(0.05 \pm 0.01 \mathrm{~s}, n=17 ; p<0.034)$ (Fig. $3 F)$.

Together, these results indicate that over development, visually evoked activity in tectal neurons becomes faster and the cell's initial response becomes less variable. Likewise, the onset latency of visual responses between cells becomes increasingly timelocked, indicating a greater degree of neural synchrony over development. These changes are disrupted by dark-rearing and may require activation of NMDARs. One possibility is that changes in response onset latency reflect maturation of the retinotectal synapses, while changes in onset differences between cells also reflects maturation of intratectal synapses. Because NMDAR activation is only one mechanism affecting development of intratectal circuits (Pratt et al., 2008) but may be more important for development of retinotectal synapses, it is possible that MK-801 had a larger effect on overall onset latencies than it did on latency differences between cells.

\section{Development of the reliability of neural response}

Over development, the visual responses of a given cell were also found to become less variable. We tested trial-to-trial variability of single cells across trials as an indicator of response reliability across experimental conditions. This was done by calculating trial-to-trial correlation values for each cell (Fig. 4A). Trial-totrial correlation is defined as the zero-lag correlation coefficient between two recordings of the same cell's response to the same stimuli across different trials (for details, see Materials and Methods, above). A larger trial-to-trial correlation value indicates less variable and more reliable neural responses. Overall, we observed a large increase of the mean trial-to-trial correlation value between stage 46 and stage 48/49 tadpoles (stage 46: $0.062 \pm 0.0152$, $n=9$; stage 48/49: $0.554 \pm 0.080 \mathrm{~s}, n=14 ; p<0.0001$ ) (Fig. $4 B$ ). Dark-reared animals showed a small mean trial-to-trial correlation value, similar to that of stage 46 tadpoles $(0.07 \pm 0.027, n=$ $17 ; p<0.0001$ ) (Fig. $4 B$ ), further supporting the hypothesis that visual input is critical for developing reliable tectal responses. NMDA receptor antagonist MK-801 also interfered with the normal developmental increase of trial-to-trial correlation values $(0.22 \pm 0.036, n=17 ; p=0.0013)$ (Fig. $4 B$ ).

In addition to being more reliable, visual responses tended to become more robust over development as the retinotectal syn-

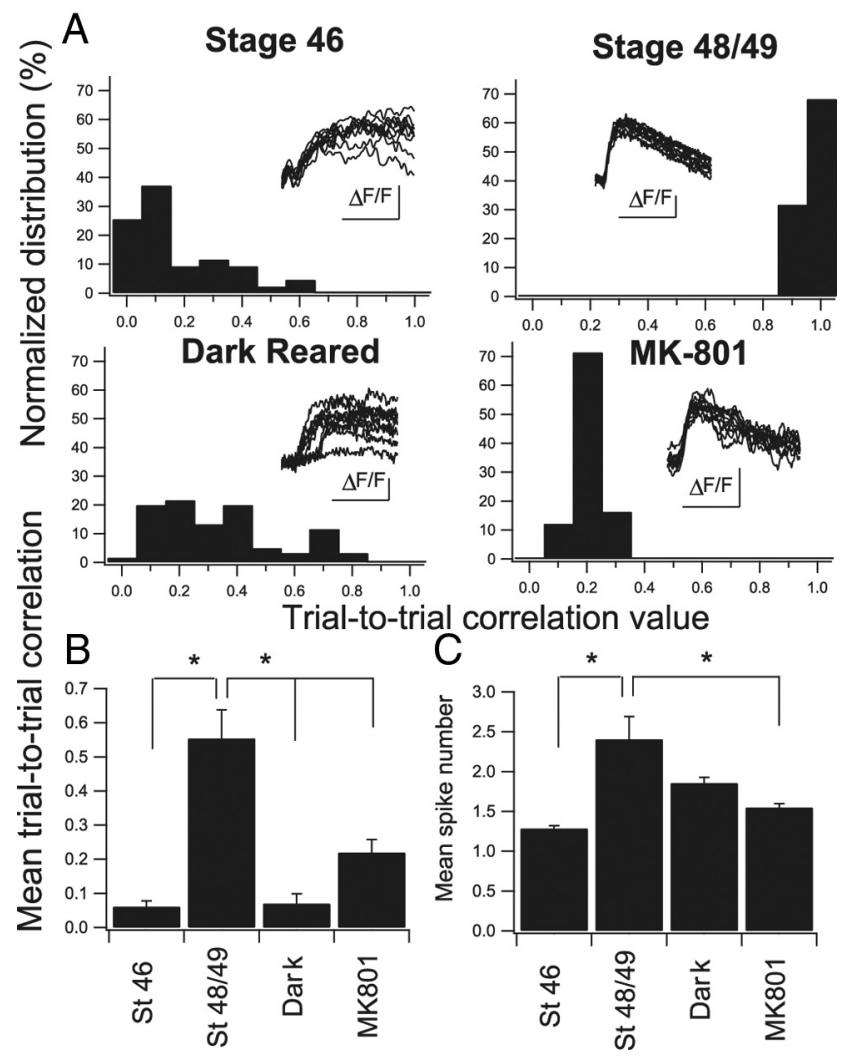

Figure 4. Developmental increase in response reliability in single neurons is activitydependent. $A$, Distribution of the trial-to-trial correlation values from single neurons. One example is shown for each experimental group. Inset, Raw fluorescence traces in response to multiple trials in a single neuron. Note the decrease in waveform variability in the older tadpoles and its dependence on neural activity. $\boldsymbol{B}$, Averaged mean trial-to-trial correlation values for each experiment in each experimental group. $\boldsymbol{C}$, Averaged mean evoked spike number during a visual response for each experiment in each experimental group. Notice that single-cell responses become less variable and more robust over development. ${ }^{*} p<0.05$; see text for exact $p$ values and number of experiments. Error bars are SEM. St, Stage.

apses strengthened. We used the number of visually evoked spikes as an indicator of the strength of the retinotectal projection. Cells from stage 48/49 animals fired significantly more spikes than cells from stage 46 animals (stage 46: 1.29 $\pm 0.03, n=$ 9; stage 48/49: $2.41 \pm 0.27, n=14 ; p=0.0013$ ) (Fig. $4 C$ ) in response to the onset of the visual stimuli. Dark-reared animals and MK-801-treated animals both responded with less spikes when compared with stage 49 controls (dark-reared: $1.86 \pm 0.07$, $n=17 ; p=0.07$; MK-801-treated: $1.55 \pm 0.05, n=17 ; p=$ 0.0086) (Fig. $4 C$ ), although the effect did not quite reach significance in the dark-reared group. This may be due to homeostatic compensation of intrinsic excitability during dark rearing. Together, these data indicate that visual input and NMDAR activation may be important for the strengthening of the retinotectal projection over development.

\section{Development of cross-correlation of spontaneous neural activity}

The correlation of evoked neural responses may be due to both feedforward retinotectal projections and to recurrent local interactions within the tectum. In our previous experiments (Fig. 1), it was not possible to distinguish between the two (see Discussion, below). To test whether local tectal circuits are involved in mediating the developmental increase in correlated activity between cells, we used an isolated whole-brain preparation. Here, tadpole 
brains were removed and directly placed on the recording dish, isolated from all sensory input (Pratt and Aizenman, 2007). During each experiment, a group of 30-90 fluorescently labeled tectal neurons was imaged over 10 trials, each lasting 16 s. Cells were included for analysis if they exhibited fluorescence transients in $>40 \%$ of trials. In the isolated brain preparation, spontaneous activity was low and therefore overall correlation values were low (Fig. $5 A, B$ ). We did not observe a developmental increase in the absolute levels of spontaneous activity across stages. However, we found that between stages 46 and 48/49, there was an increase in the amount of correlated spontaneous activity (stage 46: $0.0626 \pm 0.0008, n=29$; stage 48/49: $0.0789 \pm 0.001, n=53 ; p<$ 0.0001 ) (Fig. 5) consistent with increased maturation of local tectal circuitry. Dark-rearing had no obvious effect on the development of correlated spontaneous activity (dark-reared: $0.0811 \pm 0.002, n=74 ; p=0.3965$ ) (Fig. $5 C, D$ ). One explanation for this is that the tectum is known to receive inputs from a variety of sensory modalities (Deeg et al., 2009; Hiramoto and Cline, 2009); it is possible that these nonvisual modalities are still generating sufficient patterned activity to organize local circuitry in the absence of visual input. MK-801 treatment partially prevented the developmental increase in correlated spontaneous activity (MK-801-treated animals: $0.0726 \pm 0.002, n=53$; $p=$ 0.0054 ) (Fig. 5D), consistent with its known partial blockade of plasticity in intratectal synapses (Pratt et al., 2008).

\section{Developmental changes in latency of retinotectal inputs}

Next, we tested whether changes in the output of RGCs could contribute to the observed changes in neural synchrony observed in the tectum. If the firing pattern of RGCs becomes more synchronous over development, this would also affect the amount of evoked correlated activity in the tectum. To measure RGC output, we performed in vivo whole-cell voltage-clamp recordings from tectal neurons and measured the variability in the onset latency of the visually evoked synaptic response (Fig. 6A, $B$ ). In contrast to the calcium signal, which reflects tectal cell spiking and thus the integration of multiple synaptic inputs, the onset of the synaptic response better represents the output latencies of RGCs. Since tectal cells receive inputs from multiple RGCs, increased variability in the synchrony of the firing pattern of RGCs will be reflected as increased variability in the onset of the synaptic responses recorded in the tectum across trials. We found no differences between stages 46 and 49 tadpoles in the average onset latencies (stage 46: $129.3 \pm 10.5 \mathrm{~ms}, n=22$; stage 49: $138.1 \pm 8.8$ $\mathrm{ms}, n=13 ; p=0.213$ ) (Fig. $6 C$ ), trial-to-trial variability (average SD of onset latency across trials, stage $46: 48 \pm 5.5 \mathrm{~ms}$; stage 49: $58.5 \pm 9.6 \mathrm{~ms} ; p=0.314$ ), and the variability of the average onset latencies across cells $(p=0.113$, two-sample $F$ test for equal variance). Thus, these data suggest that changes in tectal neuron synchrony across development are unlikely to result from changes in RGC output.

\section{Computational modeling of experimental results}

Our experimental results suggest that developmental increases in correlated activity among tectal neurons requires visual activity and is in part dependent on NMDAR activation. Prior studies have implicated that NMDAR-dependent STDP in retinotectal synapses and in local tectal synapses may be important for mediating the maturation of these circuits (Zhang et al., 1998; Pratt et al., 2008) and our findings are consistent with these claims. We tested whether STDP driven by patterned visual activity could be sufficient to organize the maturation of these circuits in a way consistent with our experimental data. We created a computa-
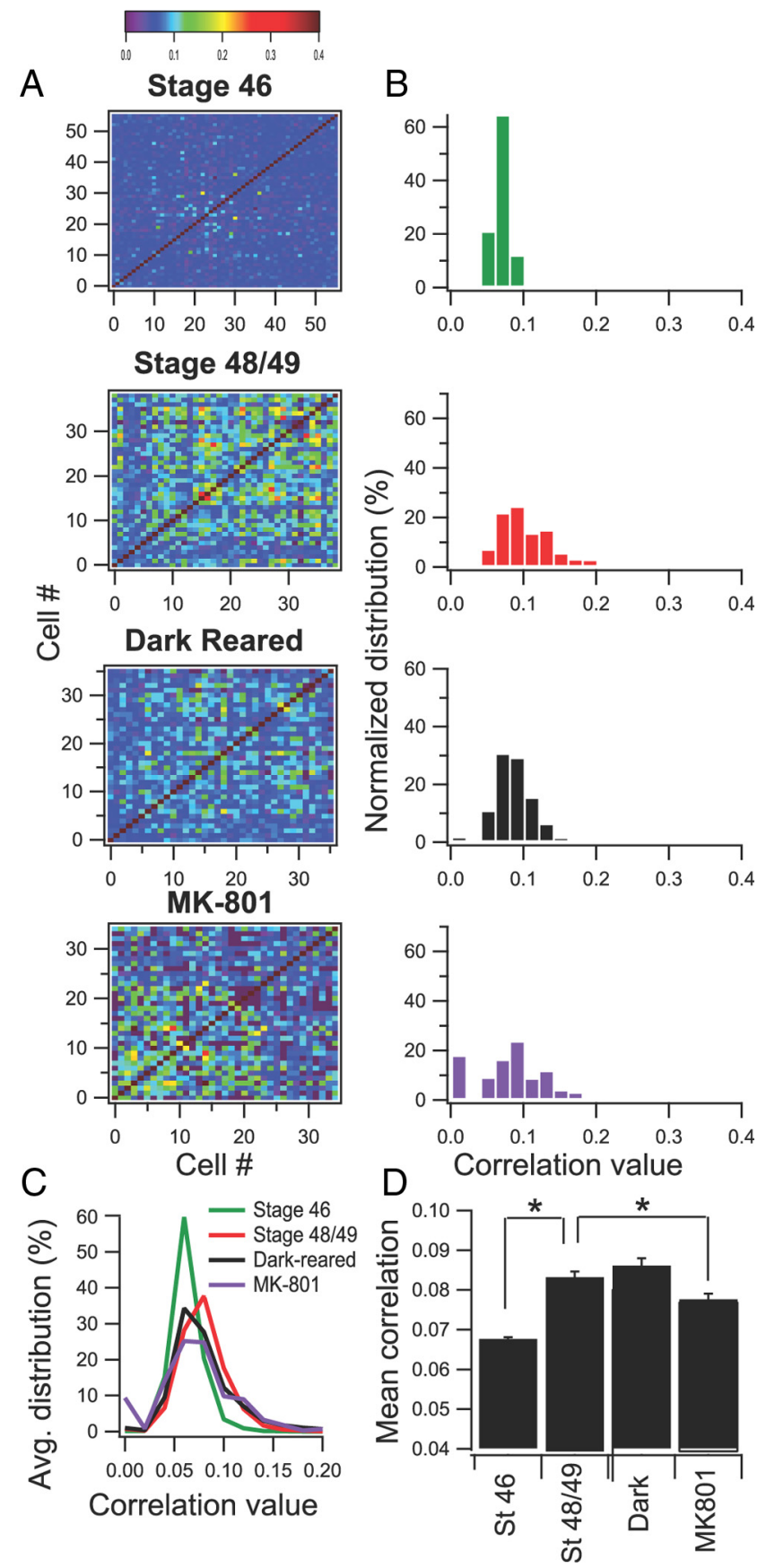

Figure 5. Developmental increase in correlated spontaneous neural activity among large groups of tectal neurons is dependent on NMDAR activation but not visual experience. $\boldsymbol{A}$, Correlation matrix resulting from spontaneous neural activity for pairs of tectal neurons that were bulk labeled with $\mathrm{Ca}^{2+}$ indicator. Colors represent average correlation values for each cell pair. One sample experiment is shown for each experimental condition. $\boldsymbol{B}$, Normalized distribution of correlation values for pairs of tectal neurons from the same examples as in $\boldsymbol{A}$. $\boldsymbol{C}$, Averaged distributions of correlation values across multiple experiments for each experimental condition. Note the small but significant shift to higher correlation values between stages 46 and 48/49. D, Average of total mean correlation value for each developmental stage and rearing condition. Although MK-801 rearing disrupted the developmental increase in correlated spontaneous activity, dark rearing did not. ${ }^{*} p<0.05$; see text for exact $p$ values and number of experiments. Error bars are SEM. St, Stage.

tional model of the retinotectal circuit consisting of a two-layer neural network (for details, see Materials and Methods, above) (Song et al., 2000; Song and Abbott, 2001). The model was comprised of an input cell layer (representing the retina) and an in- 


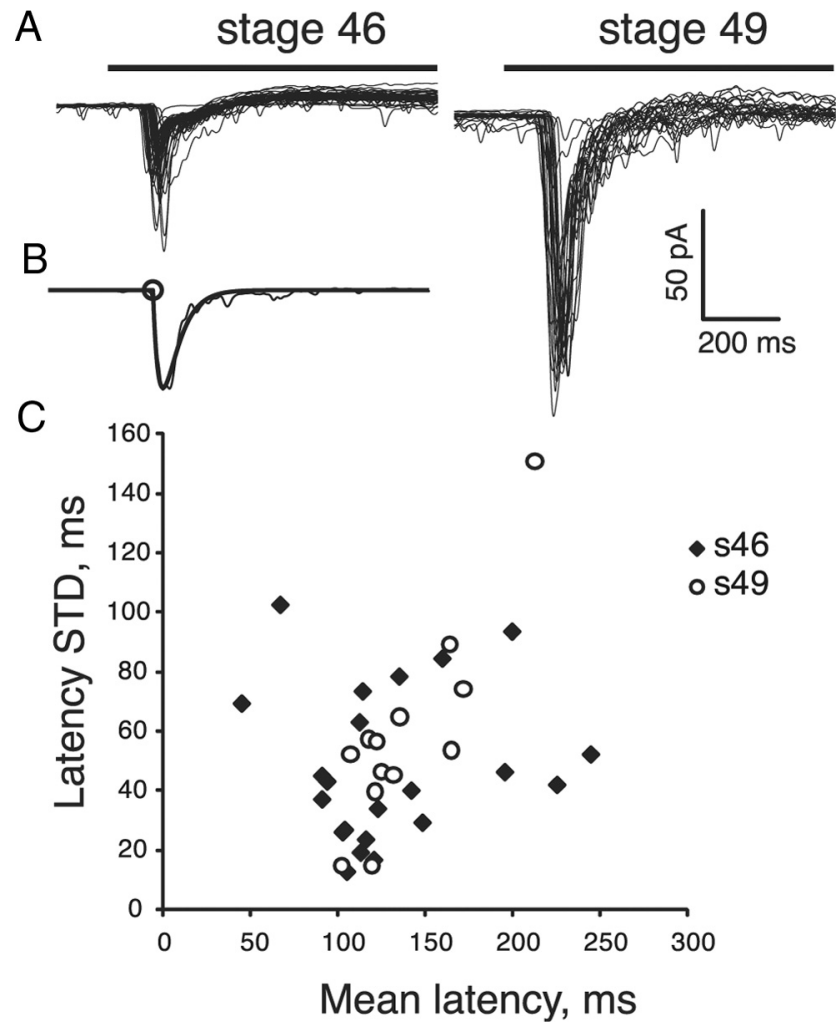

Figure 6. Retinal ganglion cell output latency and trial-to trial variability do not change over development. $\boldsymbol{A}$, Superimposed ( $n=24$ ) whole-cell voltage-clamp on responses from typical stage 46 and stage 49 tadpoles. Thick black line on the top indicates the time when visual stimulation was provided. $\boldsymbol{B}$, Typical response is shown, together with its fit and identified onset latency (marked with a circle). C, Trial-to-trial SD of latencies observed in cells from stage 46 (s46) and stage 49 (s49) tadpoles, shown versus average latencies for these cells. Note that there was no developmental change in either variability or average latency.

terconnected output cell layer (representing the optic tectum with recurrent synaptic connections), with feedforward synaptic connections between them (Fig. 7E). A STDP rule was imposed between the retinotectal synapses and between local tectal connections. Random patterned activity was generated in the input layer (see Materials and Methods, above) through several iterations. During this development period, the model reproduced most of the experimental findings observed above: increased mean cross-correlation, decreased width of the response onset latency difference distribution, and increased mean autocorrelation across trials (Fig. $7 A-C$ ). However, using the current input pattern, we did not observe an increase in the correlation values of spontaneous activity (Fig. 7D). This suggests that it may take more complex visual input patterns to cause refinement of local inputs or that a combination of patterned multisensory inputs to the tectum are required to refine local circuitry. Alternatively, refinement of local inputs may be activity-independent, although it can later be modified by STDP (Pratt et al., 2008). It should also be mentioned that recent experimental data have suggested that STDP induction parameters may be different after stage 45 , suggesting that some of the details of this model still remain to be worked out (Tsui et al., 2010).

Although this model is not the only one that can explain how activity increases correlation among tectal neurons, it does show that an STDP rule is sufficient to increase correlated activity between tectal neurons in response to patterned visual inputs.

\section{Discussion}

Using rapid multicell $\mathrm{Ca}^{2+}$ imaging in vivo, we show, for the first time, how the large-scale functional properties of optic tectal networks emerge over development, such that their output becomes increasingly spatiotemporally organized and more reliable. We further show that visual activity is critical for this process. We found that between developmental stages 46 and $48 / 49$, neurons in the tectal circuit function with increasingly higher temporal correlation, faster responses, shorter response onset latency differences, and less trial-to-trial variability in response to a whole-field visual stimulus. Increased correlation was also observed during spontaneous tectal activity in an isolated brain, suggesting that, at least in part, the functional changes are due to refinement of local tectal circuits. These developmental changes require visual activity, since dark rearing tadpoles between stages 46 and $48 / 49$ prevents this functional maturation. Chronic NMDAR blockade also partially disrupts this process. Together, these observations suggest that developmental maturation of temporal response properties of activity across the tectal network require visually driven and possibly NMDA-dependent plasticity. A simulation of the retinotectal circuit in which a STDP rule is imposed shows that this type of plasticity in combination with patterned visual input could be sufficient to organize tectal responses to light.

\section{Significance of our findings}

Our study is significant for a number of reasons. First, this is the first time that large-scale functional $\mathrm{Ca}^{2+}$ imaging using a high sampling rate (125 fps) has been used in the Xenopus tectum either in vivo or in vitro. The advantage of this method is that we can detect temporal correlations between neurons within a scale of tens of milliseconds across multiple cells. Electrophysiological recordings indicate that most recurrent activity evoked by visual inputs occurs within $500 \mathrm{~ms}$ poststimulus (Pratt et al., 2008) and that developmental changes in the temporal pattern of recurrent inputs are only observable within this time frame. Using rapid imaging, we were able to observe this temporal reorganization. Second, we answer a remaining question from the electrophysiological data of whether the changes observed in recurrent input occur in a cell autonomous manner, or whether the changes reflect an overall change in network dynamics. Our imaging data support a change in network dynamics. Third, we show that neural activity is important in this process. Although activity had been implicated in anatomical rearrangement of the retinotectal system, it was not clear whether it was necessary for the emergence of mature temporal circuit properties. Finally, our imaging method opens the door to a series of further experiments looking at the fine spatiotemporal structure of visual responses in the tectum to a variety of spatiotemporally patterned stimuli, including those that elicit visually guided behavior.

\section{Sources of correlated activity}

Which parts of the retinotectal circuit change over development? It is known that there is substantial maturation and anatomical refinement of feedforward retinotectal synapses between stages 46 and 48/49 (Cline, 1998; Debski and Cline, 2002; Ruthazer and Cline, 2004). This refinement results in smaller tectal receptive fields and sharper spatial tuning of visually driven behavior (Dong et al., 2009). Furthermore, recurrent intratectal circuits also change, resulting in shorter and less variable visual responses (Pratt et al., 2008). The large-scale changes in correlated activity across tectal neurons observed here may result from both matu- 
ration and refinement of retinotectal and intratectal synaptic circuits, and the different circuits may contribute to different properties of the responses.

Based on our method of calculating correlation, we cannot distinguish whether the increased correlation in evoked activity (Fig. 2 ) is due to maturation of retinotectal synapses alone or whether local synapses are contributing, since we cannot rule out that the increased correlation is due to elevated firing rates following a visual stimulus. Using a shift predictor (Perkel et al., 1967) based on nonmatching trials to make this distinction was not feasible in our study since we also observed a very significant decrease in trial-to-trial variability. The increase in response size over development is small compared with the very large increase in correlated activity, suggesting that it alone cannot account for the large increase in correlation, although the relation between spike rate and correlation may be nonlinear. However, the findings that the onset latency differences and trial-to-trial variability are greatly decreased over development strongly suggests that we are observing a real increase in neural synchrony over development. Furthermore, we observe an increase in correlated spontaneous activity in the isolated brain preparation that is entirely driven by local circuits. Thus, it is likely that the functional effects observed are a result of refinement at both sets of synapses.

Another possibility is that changes in synchrony may be driven, in part, by increases in correlated activity in the retina over development. However, our current whole-cell data (Fig. 6) shows that there is no difference in the trial-to-trial onset latency variability of retinotectal inputs, suggesting that the synchrony of retinal inputs to the tectum does not change significantly during these developmental stages. This suggests that developmental changes in retinal output do not contribute to the increase in tectal neural synchrony seen over development. This is consistent with results from a recent study showing that the amount of correlated activity in the retina does not change appreciably over development (Demas et al., 2011).

\section{Role of visual activity}

We found that visual activity is critical for the proper development of tectal network properties, but it remains unclear how activity mediates this developmental change. Patterned activity is important for the proper development of sensory systems across several species (Crair, 1999; Ruthazer and Aizenman, 2010). One commonly held view suggests that activity plays an instructive role during development, in which cooperative presynaptic and postsynaptic activation result in the induction of synaptic plasticity (Brown et al., 1989; Massey and Bashir, 2007). In the optic tectum, plasticity in retinotectal and intratectal synapses can be evoked by visual stimulation (Zhang et al., 1998; Dunfield and Haas, 2009), requires activation of NMDA receptors, and follows a STDP rule (Zhang et al., 2000; Engert et al., 2002; Mu and Poo,
B C

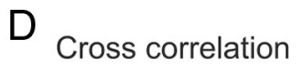

(Spont. activity)

(s) Trial-to-trial correl.
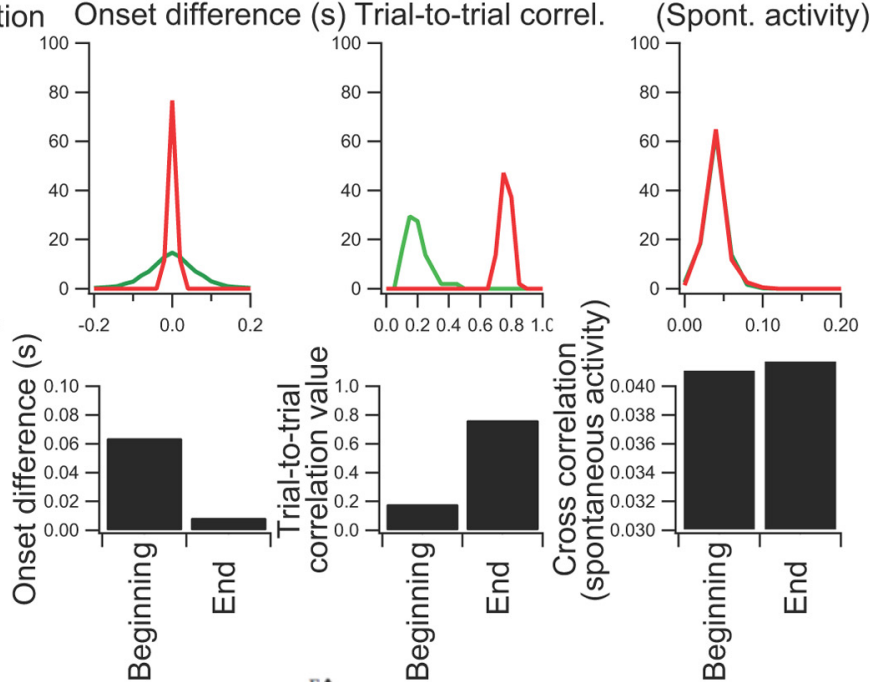

Ф

Figure 7. Developmental changes in correlation values, neural synchrony, and response reliability can be modeled using STDP rules. $\boldsymbol{A}-\boldsymbol{C}$, Distributions and mean values of cross-correlation values $(\boldsymbol{A})$, response onset latency differences $(\boldsymbol{B})$, and single cell trial-to-trial correlation (correl) values $(\boldsymbol{C})$ in response to whole-field visual stimuli at the beginning and the end of the simulation. end of the simulation. $\boldsymbol{E}$, An input cell layer and an interconnected output cell layer represent the RGCs and tectal neurons,

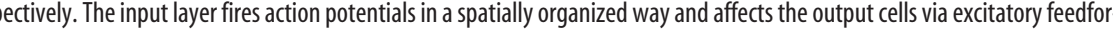
ward connections. An STDP rule was applied to all excitatory connections and a topographic organization can evolve over the simulation. LTP, Long-term potentiation; LTD, long-term depression.

2006; Pratt et al., 2008). Our data are consistent with the hypothesis that dark-rearing tadpoles between stages 46 and 48/49 blocks patterned visual activity and, by extension, plasticity in both retinotectal and intratectal synapses. This prevents activitydependent refinement of the retinotectal circuitry and therefore interferes with the normal development of the functional network properties of the tectum. Blockade of NMDAR with MK801 during this time period has a smaller effect because multiple types of synaptic plasticity may be present that can compensate. Alternatively, NMDAR blockade may interfere with development because of an overall partial reduction of neural activity, as opposed to a specific effect on neural plasticity.

\section{Potential limitations}

There are a couple of important considerations when interpreting our data. The first stems from the observation that dark rearing and MK-801 did not significantly alter the development of increased correlated spontaneous activity seen in the isolated brain preparation (Fig. 5) in contrast to its profound effect on evoked responses. One possible interpretation is that refinement of local tectal synapses may occur in a largely activityindependent manner. However, the developing tectum is known to receive significant input from nonvisual sensory modalities 
(Engert et al., 2002; Deeg et al., 2009), and the patterned activity generated by this mechanosensory input could be sufficient to organize spontaneous activity in local circuits. Thus, the effect of dark rearing becomes much more evident when the network is activated by a visual stimulus.

A second limitation in the interpretation of our data could result from the possibility that dark rearing may be primarily affecting the retinal circuitry, and therefore would disrupt the synchrony of responses observed downstream in the tectum. For example, in the mouse retina, dark rearing from birth disrupts developmental segregation of on and off layers in RGC dendrites (Tian and Copenhagen, 2003). Although we cannot fully rule out an effect of dark rearing on the Xenopus tadpole retina, we think that this is an unlikely possibility. In contrast to the mouse experiments, we begin dark rearing at stage 46 ( $~ 9 \mathrm{~d}$ postfertilization), rather than from birth, allowing significant development of the retina before dark rearing. Furthermore, in Xenopus, dark rearing does not affect the morphology of RGC dendrites (Rigel and Lom, 2004). We also did not observe a difference in the number of tectal cells preferentially responding to either on or off stimuli over development or after dark rearing.

A third issue that might affect our interpretation of the data is that we are measuring responses to whole-field visual stimuli, therefore the amount of correlated activity is expected to be higher overall than if we had used spatially restricted stimuli. Nevertheless, the observed developmental effects on correlated activity are quite striking, despite the whole-field stimulation. Using more spatially restricted stimuli, however, would probably allow us to detect spatiotemporal correlations as well as the emergence of specific groups of cells with similar response properties. Future studies with spatiotemporally controlled stimuli will allow us to understand the temporal relationships between cells with similar response properties, giving us an even more in-depth view of tectal circuit organization.

\section{Summary and future directions}

In summary, our results show that the spatiotemporal properties of the retinotectal network become increasingly robust, organized, and less variable over development. This increase in organization is driven by visual input through a mechanism that is consistent with spike-timing-dependent plasticity. This increased organization is likely to result in optimized processing of visual stimuli and visually guided behavior during development. Our experimental paradigm of rapid $\mathrm{Ca}^{2+}$ imaging in vivo will be useful for a number of future studies. For example, it will allow us to examine how a population of tectal neurons encodes behaviorally relevant visual stimuli that elicit an escape behavior (Dong et al., 2009) and contrast these to behaviorally neutral stimuli.

\section{References}

Aizenman CD, Akerman CJ, Jensen KR, Cline HT (2003) Visually driven regulation of intrinsic neuronal excitability improves stimulus detection in vivo. Neuron 39:831-842.

Akerman CJ, Cline HT (2006) Depolarizing GABAergic conductances regulate the balance of excitation to inhibition in the developing retinotectal circuit in vivo. J Neurosci 26:5117-5130.

Badea T, Goldberg J, Mao BQ, Yuste R (2001) Calcium imaging of epileptiform events with single-cell resolution. J Neurobiol 48:215-227.

Beggs JM, Plenz D (2004) Neuronal avalanches are diverse and precise activity patterns that are stable for many hours in cortical slice cultures. J Neurosci 24:5216-5229.

Brown T, Ganong A, Kairiss E, Keenan C, Kelso S (1989) Long-term potentiation in two synaptic systems of the hippocampal brain slice. In: Neural models of plasticity (Byrne JH, Berry WO, eds), pp 266-306. New York: Academic.
Cline HT (1998) Topographic maps: developing roles of synaptic plasticity. Curr Biol 8:R836-R839.

Cossart R, Aronov D, Yuste R (2003) Attractor dynamics of network UP states in the neocortex. Nature 423:283-288.

Crair MC (1999) Neuronal activity during development: permissive or instructive? Curr Opin Neurobiol 9:88-93.

Debski EA, Cline HT (2002) Activity-dependent mapping in the retinotectal projection. Curr Opin Neurobiol 12:93-99.

Deeg KE, Sears IB, Aizenman CD (2009) Development of multisensory convergence in the Xenopus optic tectum. J Neurophysiol 102:3392-3404.

Demas JA, Payne H, Cline HT (2011) Vision drives correlated activity without patterned spontaneous activity in the developing Xenopus retina. Dev Neurobiol. Advance online publication. Retrieved May 12, 2011. doi:10.1002/ dneu. 20880

Dong W, Lee RH, Xu H, Yang S, Pratt KG, Cao V, Song YK, Nurmikko A, Aizenman CD (2009) Visual avoidance in Xenopus tadpoles is correlated with the maturation of visual responses in the optic tectum. J Neurophysiol 101:803-815.

Dunfield D, Haas K (2009) Metaplasticity governs natural experiencedriven plasticity of nascent embryonic brain circuits. Neuron 64:240-250.

Engert F, Tao HW, Zhang LI, Poo MM (2002) Moving visual stimuli rapidly induce direction sensitivity of developing tectal neurons. Nature 419:470-475

Golshani P, Gonçalves JT, Khoshkhoo S, Mostany R, Smirnakis S, PorteraCailliau C (2009) Internally mediated developmental desynchronization of neocortical network activity. J Neurosci 29:10890-10899.

Hiramoto M, Cline HT (2009) Convergence of multisensory inputs in Xenopus tadpole tectum. Dev Neurobiol 69:959-971.

Ikegaya Y, Aaron G, Cossart R, Aronov D, Lampl I, Ferster D, Yuste R (2004) Synfire chains and cortical songs: temporal modules of cortical activity. Science 304:559-564.

Komai S, Licznerski P, Cetin A, Waters J, Denk W, Brecht M, Osten P (2006) Postsynaptic excitability is necessary for strengthening of cortical sensory responses during experience-dependent development. Nat Neurosci 9:1125-1133.

Maffei A, Fontanini A (2009) Network homeostasis: a matter of coordination. Curr Opin Neurobiol 19:168-173.

Massey PV, Bashir ZI (2007) Long-term depression: multiple forms and implications for brain function. Trends Neurosci 30:176-184.

Mu Y, Poo MM (2006) Spike timing-dependent LTP/LTD mediates visual experience-dependent plasticity in a developing retinotectal system. Neuron 50:115-125.

Nevin LM, Taylor MR, Baier H (2008) Hardwiring of fine synaptic layers in the zebrafish visual pathway. Neural Dev 3:36.

Niell CM, Smith SJ (2005) Functional imaging reveals rapid development of visual response properties in the zebrafish tectum. Neuron 45:941-951.

Nikolenko V, Poskanzer KE, Yuste R (2007) Two-photon photostimulation and imaging of neural circuits. Nat Methods 4:943-950.

Perkel DH, Gerstein GL, Moore GP (1967) Neuronal spike trains and stochastic point processes. II. Simultaneous spike trains. Biophys J 7:419-440.

Pratt KG, Aizenman CD (2007) Homeostatic regulation of intrinsic excitability and synaptic transmission in a developing visual circuit. J Neurosci 27:8268-8277.

Pratt KG, Dong W, Aizenman CD (2008) Development and spike timingdependent plasticity of recurrent excitation in the Xenopus optic tectum. Nat Neurosci 11:467-475.

Ramdya P, Reiter B, Engert F (2006) Reverse correlation of rapid calcium signals in the zebrafish optic tectum in vivo. J Neurosci Methods 157:230-237.

Rigel R, Lom B (2004) Xenopus laevis retinal ganglion cell dendritic arbors develop independently of visual stimulation. Impulse 1:51-58.

Rodgers JL, Nicewander WA (1988) Thirteen ways to look at the correlation coefficient. Am Stat 42:59-66.

Ruthazer ES, Aizenman CD (2010) Learning to see: patterned visual activity and the development of visual function. Trends Neurosci 33:183-192.

Ruthazer ES, Cline HT (2004) Insights into activity-dependent map formation from the retinotectal system: a middle-of-the-brain perspective. J Neurobiol 59:134-146.

Sasaki T, Takahashi N, Matsuki N, Ikegaya Y (2008) Fast and accurate detection of action potentials from somatic calcium fluctuations. J Neurophysiol 100:1668-1676. 
Schulz DJ (2006) Plasticity and stability in neuronal output via changes in intrinsic excitability: it's what's inside that counts. J Exp Biol 209: $4821-4827$.

Song S, Abbott LF (2001) Cortical development and remapping through spike timing-dependent plasticity. Neuron 32:339-350.

Song S, Miller KD, Abbott LF (2000) Competitive Hebbian learning through spike-timing-dependent synaptic plasticity. Nat Neurosci 3:919-926.

Tao HW, Poo MM (2005) Activity-dependent matching of excitatory and inhibitory inputs during refinement of visual receptive fields. Neuron 45:829-836.

Tian N, Copenhagen DR (2003) Visual stimulation is required for refinement of ON and OFF pathways in postnatal retina. Neuron 39:85-96.
Tsui J, Schwartz N and Ruthazer ES (2010) A developmentally sensitive period for spike-timing dependent plasticity in the retinotectal projection. Front Synaptic Neurosci 2:13.

Yaksi E, Friedrich RW (2006) Reconstruction of firing rate changes across neuronal populations by temporally deconvolved $\mathrm{Ca}^{2+}$ imaging. Nat Methods 3:377-383.

Yuste R, Peterlin Z, Kozloski J (2000) Optical probing: a novel technique for revealing neuronal circuits. J Physiol 526[Suppl]:20s.

Zhang LI, Tao HW, Holt CE, Harris WA, Poo M (1998) A critical window for cooperation and competition among developing retinotectal synapses. Nature 395:37-44.

Zhang LI, Tao HW, Poo M (2000) Visual input induces long-term potentiation of developing retinotectal synapses. Nat Neurosci 3:708-715. 